\title{
Comparativo de inserción a estadías del nivel TSU en Turismo de la UTRM entre el modelo BIS y tradicional
}

\section{Comparison of insertion to stays of the TSU level in Tourism of the UTRM between the BIS and traditional model}

\author{
GÚADARRAMA-GÓMEZ, Irma†*, LARIOS-CALDERÓN, Aralí y BALDERAS-ELORZA, Carlos \\ Raymundo
}

Universidad Tecnológica de la Riviera Maya

ID $1^{\mathrm{er}}$ Autor: Irma, Gúadarrama-Gómez / ORC ID: 0000-0003-4668-9432, CVU CONACYT ID: 472108

ID $1^{\mathrm{er}}$ Coautor: Aralí, Larios-Calderón / ORC ID: 0000-0002-2136-1118, CVU CONACYT ID: 473109

ID $2^{\text {do }}$ Coautor: Carlos Raymundo, Balderas-Elorza / ORC ID: 0000-0002-8069-4043, CVU CONACYT ID: 472108

DOI: $10.35429 / J E S C .2019 .7 .3 .21 .30$

Recibido: 02 de Enero, 2019; Aceptado 30 de Marzo, 2019

\begin{abstract}
Resumen
Objetivo: Con la finalidad de dar seguimiento a la investigación sobre la inserción de los alumnos del programa de TSU en Turismo área Hotelería al proceso de estadías publicada en el 2017, se realizó un análisis comparativo de la inserción a las prácticas profesionales entre los estudiantes del nivel TSU área Turismo de la UTRM, del modelo tradicional y el modelo BIS, para identificar el aporte en la formación del recurso humano de la Institución hacia el sector hotelero. Metodología: El presente estudio es del orden cualitativo, con un alcance descriptivo, considerando los resultados obtenidos en la primera investigación, y posteriormente contrastándolos con los resultados de la inserción a estadías de los alumnos generación 2017 - 2019 inscritos en el modelo BIS (Bilinguie, Internacional y Sustentable). Para realizar el comparativo se tomaron en cuenta los factores: socioeconómico, departamento y/o área de inserción. Contribución: Los resultados serán referente para las UUTT y PP que ofrecen el modelo BIS dentro del programa de Turismo, permitiéndoles identificar la formación del recurso humano que está aportando el nuevo modelo en comparación al anterior, dentro del sector hotelero.
\end{abstract}

Modelo BIS, Estadías, Comparativo.

\begin{abstract}
Objective: In order to follow up on the research published in 2017 regarding the insertion of TSU Tourism: Hotel Area students in the process of the professional practicum within the productive sector, a comparative analysis of the traditional modality and the Bilingual, International and Sustainable (BIS) modality was carried out to identify the contribution from the students in the formation of human resources of the institution towards the hospitality industry. Methodology: This was a qualitative study, with a descriptive scope. The results obtained from the first investigation were considered and contrasted with the results of the insertion in professional practicums of the generation of 2017 - 2019 BIS modality. The factors considered in the comparison were: socioeconomic, company and area of insertion. Contribution: The results will be a reference for the Subsystem of Technological and Polytechnic Universities that impart the BIS modality in the Tourism program. This will allow them to identify the formation of the human resource by students in the BIS modality compared to what was seen in the traditional model, within the hospitality sector.
\end{abstract}

BIS Model, Professional Practices, Comparative

Citación: GÚADARRAMA-GÓMEZ, Irma, LARIOS-CALDERÓN, Aralí y BALDERAS-ELORZA, Carlos Raymundo. Comparativo de inserción a estadías del nivel TSU en Turismo de la UTRM entre el modelo BIS y tradicional. Revista de Ciencias de la Educación. 2019. 3-7: 21-30

$\dagger$ Investigador contribuyendo como primer autor. 


\section{Introducción}

Las prácticas profesionales constituyen un mecanismo destinado a completar y perfeccionar el proceso formativo de los alumnos. Éstas, pueden ser vistas como un reflejo de las capacidades adquiridas por los estudiantes durante su proceso de formación profesional dentro de la Universidad. En la Universidad Tecnológica de la Riviera Maya, las prácticas profesionales se llevan a cabo en el último ciclo escolar como cierre de la formación adquirida en el proceso enseñanza - aprendizaje de cada una de las asignaturas del programa, siendo éstas un requisito de titulación.

Actualmente, dentro de la Universidad, no hay un seguimiento de los resultados del proceso de estadía que sea un parámetro del quehacer formativo de la Universidad hacia a los alumnos y menos aún, un análisis de la implementación del nuevo programa educativo llamado modelo Bilingüe Internacional $\mathrm{y}$ Sustentable (BIS), por lo que con la finalidad de dar seguimiento a la investigación realizada en el 2017 sobre la inserción de los alumnos del programa de TSU en Turismo área Hotelería al proceso de estadías y hacer un comparativo de esos resultados con los resultados de inserción de los alumnos del nuevo modelo de estudios (BIS), es que se lleva a cabo la presente investigación faculta identificar el área departamental donde el practicante se ha insertado, permitiendo al mismo tiempo hacer una evaluación de los resultados del modelo educativo actual en relación a la aportación de la formación del recurso humano que está teniendo la Universidad específicamente en el sector hotelero de Playa del Carmen.

Para tal efecto, en este estudio se identifican los antecedentes de La Universidad Tecnológica de la Riviera Maya, así como del Modelo BIS, en un segundo apartado, se habla del proceso metodológico comparativo utilizado en la presente investigación, y finalmente se enuncian los resultados de este.

\section{Playa del Carmen y su actividad turística en números}

Playa del Carmen, es uno de los destinos turísticos más importantes de México, y uno de los más visitados a nivel mundial. Pertenece al denominado corredor turístico Riviera Maya, el cual tuvo una afluencia turística de 6, 211, 454 turistas en el 2018.
El gasto promedio de los turistas en ese mismo año fue de $\$ 1,095.80$ dólares, generando una derrama de $\$ 6,806.51$ MDD y una diferencia positiva de $6.2 \%$ en comparación al año anterior. (SEDETUR, 2018) En cuanto a su infraestructura hotelera, Playa del Carmen cuenta con 271 hoteles, y 39, 845 cuartos de hotel. La ocupación promedio en el 2018 fue de $81.7 \%$, un $0.8 \%$ menor al año 2017. En relación con la estadía promedio, de igual manera disminuyó, de estar en 6.1 noches, paso al 6.0, representando un $-0.1 \%$. (SEDETUR, 2018)

\section{Contexto económico social de Playa del Carmen}

Playa del Carmen, es la cabecera municipal del municipio de Solidaridad, siendo su principal actividad económica el turismo. La aceleración económica de esta actividad ha traído consigo la migración de personas de todas partes del mundo, pero principalmente de los estados de Yucatán, Veracruz, Tabasco, Distrito Federal y Chiapas (Gobierno del Estado de Quintana Roo). Desde finales de los años 90's, "el crecimiento social ha causado desequilibrios en la infraestructura urbana, económica, medio ambiental y en general, en la atención del rezago social" (Torres \& Rojas , 2015)

En 1960 de acuerdo con los datos del Censo de Población de INEGI, la localidad conocida como Playa del Carmen contaba con 93 habitantes, y ya para el año de 1970 contaba con una población de 232 habitantes, lo que representó una tasa media anual del 9.57 por ciento. En 1980 Playa del Carmen había alcanzado un total de 737 habitantes (una tasa anual del $12.25 \%$ en el periodo $1970-1980$ ), para el año de 1990 en Playa del Carmen residían 3,098 habitantes, es decir, tuvo un incremento de $15.44 \%$, con 7 años de fundación el municipio crecía a una tasa de media anual de $24.7 \%$ es no sólo de las más grandes del estado, sino a nivel nacional, en el año 2000 ya contaba con una población de 47,757, en el 2010 contaba con 149,923 personas (Camara 2007), y para el año 2014 según estadísticas poblacionales del INEGI Playa del Carmen cuenta con 206,716 habitantes.

El incremento poblacional en la región es una situación que se agudiza principalmente por la construcción de infraestructura hotelera, la cual atrae a un importante número de personas en busca de trabajo.

GÚADARRAMA-GÓMEZ, Irma, LARIOS-CALDERÓN, Aralí y BALDERAS-ELORZA, Carlos Raymundo. Comparativo de inserción a estadías del nivel TSU en Turismo de la UTRM entre el modelo BIS y tradicional. Revista de Ciencias de la Educación. 2019 
Sin embargo, el índice migratorio registrado en los últimos años y la creación de empleos por el desarrollo de infraestructura turística han derivado en graves carencias de infraestructura para dar respuesta a la creciente y permanente demanda de los nuevos pobladores, por lo que los servicios existentes, ante la falta de recursos propios y externos, se han vuelto obsoletos (ibid). (Herrera, s/f, p. 29)

\section{Recurso Humano en la industria hotelera de Playa del Carmen}

La Riviera Maya se ha convertido en uno de los polos turísticos más importantes de México. En poco más de 20 años, se ha pasado de 1.500 habitaciones de hotel a principios de los años 90's, a más de 40,000 habitaciones en la actualidad. Este rápido desarrollo del sector ha generado una fuerte demanda de trabajadores calificados, que los sistemas formativos tradicionales difícilmente podrían satisfacer: sólo el año pasado se crearon 13.000 nuevos empleos. (Asociación de Hoteles de Riviera Maya, 2015)

Los puestos de mayor demanda por el sector hotelero son: camarista, mesero, áreas públicas, stewards, cocina, recepción, garrotero, ayudante de cantinero, mantenimiento $y$ operador de cuartos. En relación con los puestos de menor rotación se encuentran: administración, recepción, camarista, mantenimiento, mesero, ventas, ama de llaves, telefonista, auxiliar de lavandería y cocina. A su vez, los puestos más difíciles de encontrar son: mesero, camarista, recepción, concierge, auxiliar de limpieza, tabla roquero, encargado de planta de tratamiento y sous chef. (Asociación de Hoteles de Riviera Maya, 2015)

\section{Antecedentes del modelo Bilingüe, Internacional y Sustentable en las Universidades Tecnológicas}

En México, dentro del contexto educativo se han generado nuevos retos relacionados con los temas de sustentabilidad, así como lo concerniente con los avances tecnológicos y científicos que se presentan en la sociedad, derivados de una inminente globalización. Lo anterior, ha llevado a generar estrategias que permitan promover un desarrollo y crecimiento orientado a la competitividad internacional y cumplir con ello, las expectativas que demanda el actual contexto social y económico del país.
Es importante hacer mención que, tanto el Plan Nacional de Desarrollo como el Programa Sectorial de Educación señalan expresamente que, para los efectos de continuar con el desarrollo de los mecanismos tendientes al aseguramiento de la calidad de los programas e instituciones de educación superior, se deberá apoyar el surgimiento de nuevos modelos de Formación Profesional que atiendan el imperativo de internacionalizar la educación superior. Ante tal compromiso es que se impulsa un nuevo Modelo de Universidad Tecnológica Bilingüe, Internacional y Sustentable (U-BIS), cuyo fin es coadyuvar en el esfuerzo por ampliar las alternativas para emprender el gran reto de la competitividad. (SEP, 2017)

Basado en lo anterior, la modalidad Bilingüe, Internacional y Sustentable (BIS) dentro del Subsistema de Universidades Tecnológicas y Politécnicas (SUTyP), surgió de las necesidades identificadas en el Estado de Aguascalientes, por la inversión extranjera principalmente en el sector automotriz y de desarrollo de software, la cual requiere talento humano altamente capacitado y con dominio de un segundo idioma. En 2010, el entonces Gobernador Constitucional del Estado de Aguascalientes, Ing. Carlos Lozano de la Torre, solicitó al Coordinador General de Universidades Tecnológicas y Politécnicas (UTyP's), Ing. Héctor Arreola Soria, la creación de una Universidad donde se enseñara inglés a los Técnicos Superiores Universitarios para que adquirieran habilidades en un segundo idioma y fuesen fácilmente contratados por las empresas transnacionales ubicadas en esa entidad federativa. La Universidad Tecnológica El Retoño comenzó operaciones en agosto del 2012, siendo la primera universidad del país en implementar la modalidad BIS, conservando el modelo de las UTyP's, el cual ofrece carreras basadas en las necesidades reales del sector empresarial de las entidades federativas, siendo en ese momento, la única en su tipo dentro de la educación pública en México (CGUTyP, 2017).

El objetivo general del modelo dentro del tema sustentable es proporcionar un marco de actuación para las actividades sustantivas y adjetivas de las Universidades Tecnológicas y Politécnicas BIS, que permitan disminuir la huella ecológica e incrementar la responsabilidad social para apoyar, en un marco de congruencia, el modelo de educación para la sustentabilidad. 
Por lo que respecta los ejes bilingüe e internacional, estos tienen como objetivo generar redes de intercambio cultural y de experiencias educativas, con universidades en el extranjero, así mismo fomentar la cooperación regional, ya que la movilidad debe actuar como un agente activo en el proceso de fortalecimiento de la cooperación entre IES a través del intercambio de conocimientos. Según la CGUTyP (2017) la movilidad Internacional es a través de los programas de las becas del Consejo Nacional de Ciencia y Tecnología (CONACyT) para la realización de posgrados de calidad en el extranjero, Proyecta 100,000 Estados Unidos de América, Proyecta 10,000 Canadá, becas México Francia Ingenieros Tecnología (MEXFITEC), MEXPROTEC, y la Beca de Movilidad Internacional los que han permitido esta vinculación internacional.

Aunque actualmente existen 119 Universidades Tecnológicas y 62 Politécnicas en el país, no todas han migrado al nuevo modelo BIS. Actualmente, 41 Universidades Tecnológicas y Politécnicas en México operan su sistema de enseñanza aprendizaje en inglés bajo el modelo BIS. (ver figura 1).

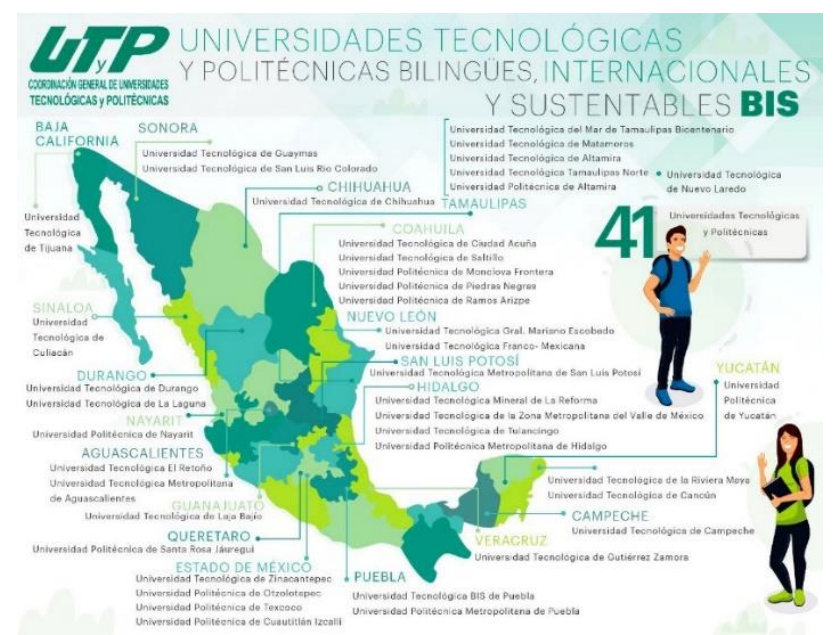

Figura 1 Universidades Tecnológicas y Politécnicas Bilingües, Internacional y Sustentables en México Fuente: Universidad Tecnológica de la Riviera Maya (2019)

La CGUTyP (2018), en su marco de referencia hace mención que "la Modalidad BIS del Subsistema, propone además de una formación técnica, una educación bilingüe de calidad, inclusiva y equitativa orientada a la generación de un nuevo modelo civilizatorio que proponga soluciones inteligentes e innovadoras a los problemas más grandes del mundo para mejorar la calidad de vida de todas las personas.
Mediante la formación integral de ciudadanos globales, responsables y profesionistas competentes, basados en el desarrollo humano, el bilingüismo y la internacionalización; con una sólida identidad nacional y una visión global que posibilite su resiliencia y excelente desempeño en un entorno global altamente competitivo, contribuyendo con responsabilidad social al desarrollo nacional." Lo anterior se suma a su modelo pedagógico tradicional basado en competencias profesionales, que se caracteriza por su sistema escolarizado y cuatrimestral, generando un desarrollo integral en los tres saberes (saber, haber hacer y saber ser).

\section{Modelo BIS, Universidad Tecnológica de la Riviera Maya (área Turismo)}

Al implementarse el modelo BIS en la Universidad Tecnológica de la Riviera Maya (UTRM) en el año 2017, la modificación en el programa de turismo consistió en la actualización de la estructura de los cuatrimestres, incorporando al inicio, un cuatrimestre de formación propedéutica de inmersión total al segundo idioma, llamado introducción a la lengua extranjera, con una duración de 525 horas, con la finalidad de homologar a los alumnos de nuevo ingreso en el nivel A2. Además, en el primer cuatrimestre de formación técnica, se imparten dos materias en idioma extranjero, y cuatro en el segundo cuatrimestre. A partir del tercero todas las asignaturas de la carrera se imparten en inglés. (ver figura 2).

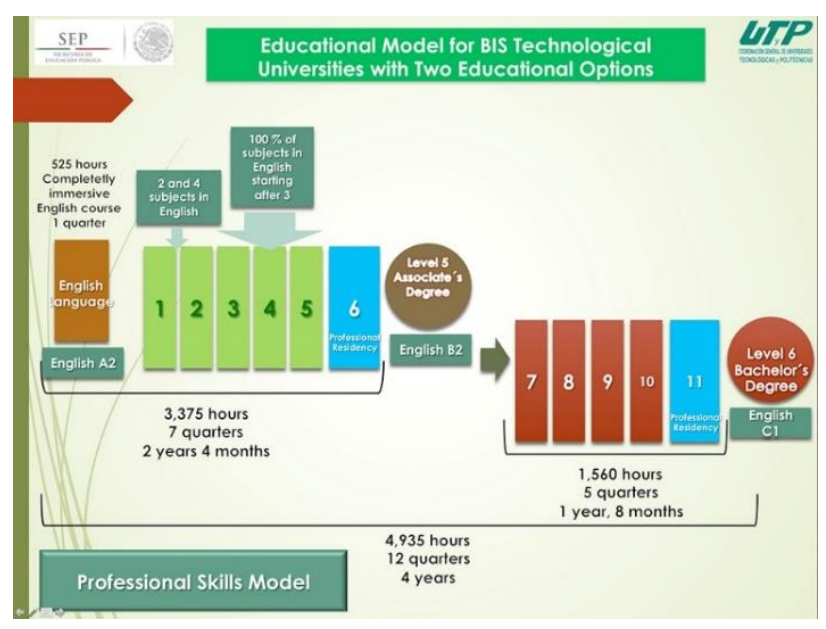

Figura 2 Modelo Educativo BIS

Fuente https://www.utrivieramaya.edu.mx/utrm1/modeloeducativo 
Con respeto a la duración del programa en número de cuatrimestres, este se mantiene con una duración de dos años divididos en, seis cuatrimestres para el TSU y un año y ocho meses más para licenciatura.

En relación con el proceso de enseñanza aprendizaje, se continúa con el enfoque por competencias profesionales planteado en Balderas, Larios y Guadarrama, 2017.

La finalidad del modelo BIS es el dominio del idioma inglés como principal habilidad profesional necesaria en los alumnos de acuerdo con su entorno, por lo que el modelo contempla diferentes metas en el idioma inglés (Ver tabla 1).

\begin{tabular}{|c|c|c|}
\hline Cuatrimestre & $\begin{array}{l}\text { Nivel inglés } \\
\text { (MCERL) }\end{array}$ & Ciclo de Formación \\
\hline $\begin{array}{l}\text { Inmersión a la } \\
\text { Lengua } \\
\text { Extranjera }\end{array}$ & $\mathrm{A} 2$ & \multirow[t]{7}{*}{$\begin{array}{l}\text { Técnico Superior } \\
\text { Universitario } \\
\text { Profesional } \\
\text { Asociado }\end{array}$} \\
\hline $1^{\circ}$ & A2 & \\
\hline $2^{\circ}$ & A2 & \\
\hline $3^{\circ}$ & B1 & \\
\hline $4^{\circ}$ & B1 & \\
\hline $5^{\circ}$ & B1 & \\
\hline $6^{\circ}$ & $\mathrm{B} 1 / \mathrm{B} 2$ & \\
\hline $7^{\circ}$ & $\mathrm{B} 1 / \mathrm{B} 2$ & \multirow{5}{*}{$\begin{array}{l}\text { Ingeniería } \\
\text { Licenciatura }\end{array}$} \\
\hline $8^{\circ}$ & B2 & \\
\hline $9^{\circ}$ & $\mathrm{B} 2$ & \\
\hline $10^{\circ}$ & $\mathrm{B} 2 / \mathrm{C} 1$ & \\
\hline $11^{\circ}$ & $\mathrm{B} 2 / \mathrm{C} 1$ & \\
\hline
\end{tabular}

Tabla 1 Nivel de Dominio de la lengua por cuatrimestre y ciclo de formación

Fuente: Elaboración Propia con base en los lineamientos de la CGUTyP

En cuanto al perfil general de egreso del estudiante, este sigue siendo el mismo, a diferencia de que, en el modelo anterior, se tenía detallado el perfil de egreso por grado obtenido (TSU y Licenciatura).

Es de destacar, que la incorporación de información al modelo BIS es en relación con el perfil y funciones de los docentes, siendo esta: El perfil de los maestros, su interacción con autoridades y alumnos, así como su organización, son cuestiones fundamentales que determinarán en buena medida, el desarrollo de la Institución. Al ingresar el profesor a la Universidad debe tener como mínimo el grado de licenciatura, experiencia en la industria, laborar en ese momento en alguna empresa y poseer experiencia docente de acuerdo con la disciplina que va a impartir.

ISSN: 2523-2436

ECORFAN® Todos los derechos reservados
Entre las principales funciones que debe desempeñar, además de impartir clases, está el desarrollar su método de enseñanza con énfasis principal en la práctica del alumno fomentar el autoaprendizaje, elaborar material didáctico, etc. También es muy importante que promueva la identificación de las empresas donde el alumno va a realizar su estadía. Por último, el docente debe estar preparado y dispuesto para realizar estudios y servicios tecnológicos para los organismos que así lo requieran, así como para la propia Universidad (UTRM, 2019)

Por lo anterior, se puede apreciar que el idioma ingles no forma parte ni del perfil, ni de las funciones del docente, sin embargo, la capacitación en el idioma inglés se comenzó antes de la implementación del modelo BIS y a la fecha, es obligatorio obtener el nivel B2 para impartir clases en el modelo.

\section{Metodología}

Con la finalidad de dar seguimiento a la investigación sobre la inserción de los alumnos del programa de TSU en Turismo área Hotelería al programa de estadías publicada en el 2017, esta segunda parte consistió en realizar un análisis comparativo de la inserción a las prácticas profesionales dentro del sector productivo de los estudiantes de turismo del nivel TSU área Turismo de la UTRM entre el modelo tradicional y el modelo BIS, para identificar el aporte en la formación del recurso humano de la Institución hacia el sector hotelero.

El presente estudio es del orden cualitativo, ya que cubre una serie de métodos y técnicas con valor interpretativo que pretende describir, abalizar, descodificar, traducir y sintetizar el significado de hechos que se suscitan más o menos de manera natural, según lo mencionado por Maanen citado en Álvarez (2014). El alcance de la investigación es descriptivo, pues busca especificar los perfiles de las personas y es útil para mostrar con precisión las dimensiones de un determinado fenómeno. Para el presente estudio, se consideró de manera discrecional a la totalidad de alumnos que terminaron el Técnico Superior Universitario en Turismo y que continuaron con los estudios de Licenciatura en Gestión y Desarrollo Turístico en la Universidad Tecnológica de la Riviera Maya, por lo que según Battaglia citado en Hernández (2014) la población es de participantes voluntarios, resultando un total de 28 estudiantes.

GÚADARRAMA-GÓMEZ, Irma, LARIOS-CALDERÓN, Aralí y BALDERAS-ELORZA, Carlos Raymundo. Comparativo de inserción a estadías del nivel TSU en Turismo de la UTRM entre el modelo BIS y tradicional. Revista de Ciencias de la Educación. 2019 
La recolección de información se llevó a cabo en el aula de clases, a través del registro que cada alumno hizo en la base de datos de inserción a estadías (ver anexo 1) teniendo previamente el nombre completo del estudiante. Los campos que los alumnos registraron fueron: el nombre de la empresa en la que realizaron sus estadías, el departamento y el puesto en el que se desempeñaron, así como la localidad y el municipio. Adicional a estos datos y con la intensión de identificar el nivel del idioma inglés con el que el alumno se percibía al momento de realizar sus estadías, se colocó la descripción de los niveles de inglés de acuerdo con el Marco Común Europeo de Referencia (Instituto Cervantes, 2017), pero se identificaron con una numeración secuencial del uno al seis en lugar de escribir tácitamente los niveles A1, A2, B1, $\mathrm{B} 2, \mathrm{C} 1$ y $\mathrm{C} 2$ para que el alumno no se viera influenciado por esta información y su respuesta se diera en función a la descripción del nivel.

Posteriormente, la base de datos de inserción a estadías se complementó con el nivel de inglés certificado de cada alumno, obtenido mediante el iTEP Certificate Test (International Test of Enghlish Proficiency) al momento de terminar el nivel TSU e ingresar a la Licenciatura. Para el análisis, se ordenó la información con la finalidad de explorar los datos, organizarlos por unidades y categorías, describir los patrones y otórgales sentido para llevar a cabo el comparativo.

Los factores de análisis de TSU modelo BIS generación 2017 - 2019 fueron:

$\begin{array}{ll}\text { a) } & \text { Demográficos: } \\ - & \text { Género } \\ - & \text { Municipio } \\ \text { b) } & \text { Factores inserción al área departamental: } \\ - & \text { Departamento } \\ - & \text { Puesto desempeñado } \\ \text { c) } & \text { Factores nivel de inglés } \\ - & \text { Nivel percibido } \\ - & \text { Nivel certificado }\end{array}$

Posteriormente, se utilizó el método comparativo para confrontar los resultados de ambos estudios. El objetivo fundamental del método consiste en la generalización empírica.
Entre las ventajas que ofrece el método comparativo se cuentan el comprender cosas desconocidas a partir de las conocidas, la posibilidad de explicarlas e interpretarlas, perfilar nuevos conocimientos, destacar lo peculiar de fenómenos conocidos, sistematizar la información distinguiendo las diferencias con fenómenos o casos similares.

A continuación, se detallan de los pasos que se llevaron a cabo para realizar el comparativo entre los resultados obtenidos del estudio publicado en 2017 titulado "Comparativo de inserción del proceso de estadías o prácticas profesionales de los niveles educativos de Técnico Superior Universitario en Turismo y Licenciatura en Gestión y Desarrollo Turístico" (Balderas Elorza, Larios Calderón , \& Guadarrama Gómez, 2017) y los resultados del análisis de inserción de la generación 2017 2019 del programa BIS, dado que las variables no eran semejantes.

La primera etapa consistió en la configuración de una estructura teórica donde se definieron las propiedades y características de los casos a comparar y que permitiera una clasificación que identifique las variaciones y semejanzas del objeto de estudio.

En una segunda etapa, se definieron los criterios o factores asumidos para la selección de la muestra, es decir de los casos a elegir objeto de estudio. En este sentido se consideró justificar la selección cuidando que los casos resultantes efectivamente comparables y relevantes (ver tabla 2).

Con base en lo anterior, se procedió al análisis a partir de la contrastación de los factores determinados en la tabla 2.

\begin{tabular}{|l|l|}
\hline \multicolumn{2}{|c|}{ Factor } \\
\hline Demográfico & $\begin{array}{l}\text { Este factor está representado por } \\
\text { las características de género del } \\
\text { practicante. }\end{array}$ \\
\hline $\begin{array}{l}\text { Departamento o } \\
\text { puesto de trabajo }\end{array}$ & $\begin{array}{l}\text { Este se define como el área } \\
\text { departamental donde el } \\
\text { practicante se insertó para hacer } \\
\text { sus estadías profesionales. }\end{array}$ \\
\hline
\end{tabular}

Tabla 2 Factores de análisis comparativo entre modelo BIS y Tradicional Fuente: Elaboración Propia 


\section{Resultados}

A partir de la metodología establecida se detallan los resultados por factores de análisis enfocados a la primera generación TSU BIS de la UTRM, para posteriormente presentar el Comparativo de inserción a estadías del nivel TSU en Turismo de la UTRM entre el modelo BIS y tradicional.

\section{Factores de análisis de TSU modelo BIS generación 2017 - 2019.}

\section{- $\quad$ Factores Demográficos}

Respecto a los factores demográficos analizados en este estudio en relación con el género de los estudiantes objeto de estudio, se identifica igualdad en los porcentajes con un 50\% (ver gráfica 1).

\section{GÉNERO}

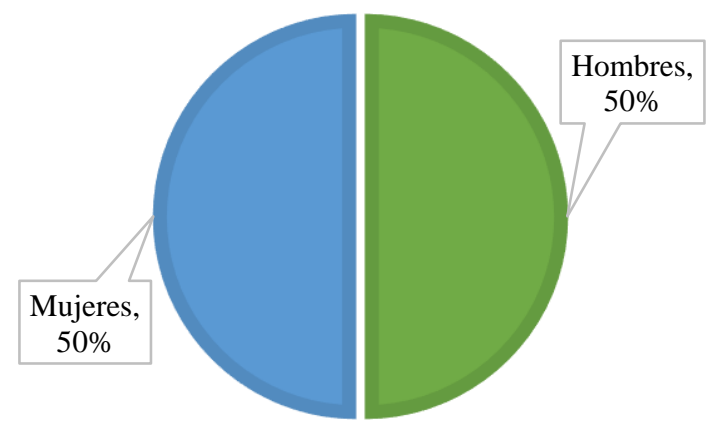

Gráfica 1 Género de los practicantes

Fuente: Elaboración Propia

En relación con la localización de las empresas donde los alumnos desarrollaron las prácticas profesionales, se identifica que el $93 \%$ se encuentran en el Municipio de Solidaridad, seguido de los Municipios aledaños de Tulum y Benito Juárez con un 4\% y 3\% respectivamente (ver gráfica 2).

\section{MUNICIPIO}

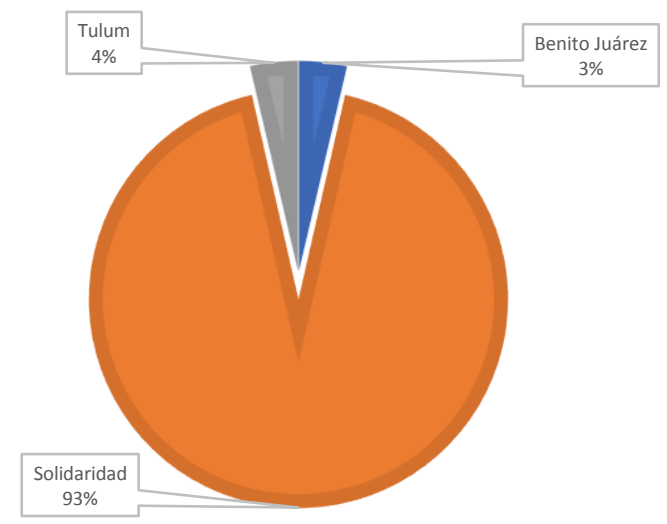

Gráfica 2 Municipios donde se llevó a cabo las prácticas profesionales

Fuente: Elaboración propia

- $\quad$ Factores inserción al área departamental.

En este apartado, se analizaron dos factores: el departamento y el puesto desempeñado. Referente al porcentaje con mayor inserción en los departamentos, recepción es el que destaca con un 29\%, seguido por los departamentos de bodas y recursos humanos, ambos con $14 \%$. Siendo animación el menos recurrente con $3 \%$. (ver gráfica 3 ).

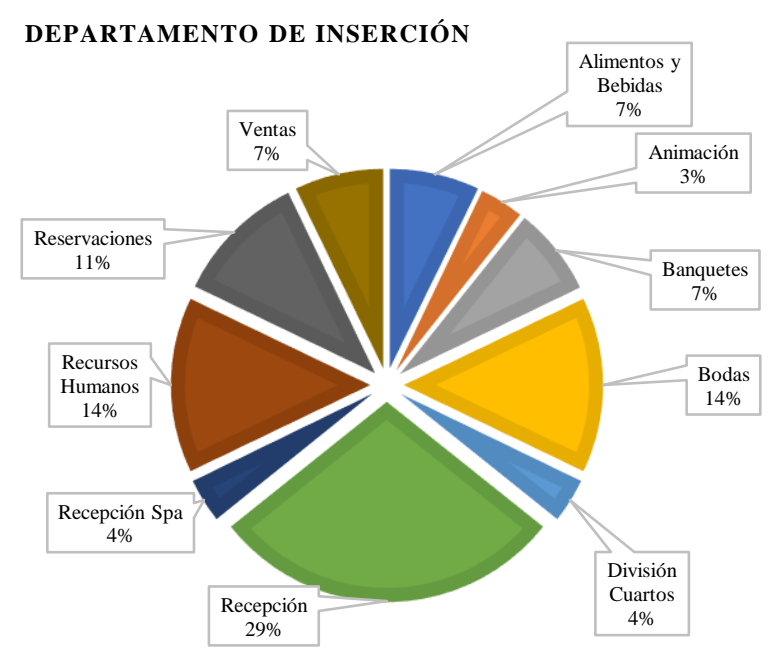

Gráfica 3 Departamentos de inserción Fuente: Elaboración Propia

Con respecto a los puestos desempeñados durante el tiempo de estadías, se observa que el $69 \%$ de los practicantes realiza funciones operativas. directamente relacionadas con la atención al cliente (Front of the house), donde se destaca los puestos de: recepcionista (32\%), asistente de bodas (14\%), mesero (7\%), hostess, reservacionista, concierge y encargado de Kid's Club con un $4 \%$ cada uno. 
Sobre los puestos vinculados al "Back of the house", también conocidos como áreas administrativas, es donde se insertó una minoría de los alumnos en estadías representando el $31 \%$, dividiéndose de la siguiente manera: capacitación (7\%), reclutamiento (4\%), auxiliar de reclutamiento selección de personal $(4 \%)$, auxiliar de reservaciones (4\%), reservacionista $(4 \%)$, asistente de gerente de reservaciones (3 $\%$ ), asistente del coordinador de bodas (3\%), asistente administrativo (3\%) y asistente de gerente de ventas (3\%) que en conjunto (ver gráfica 4).

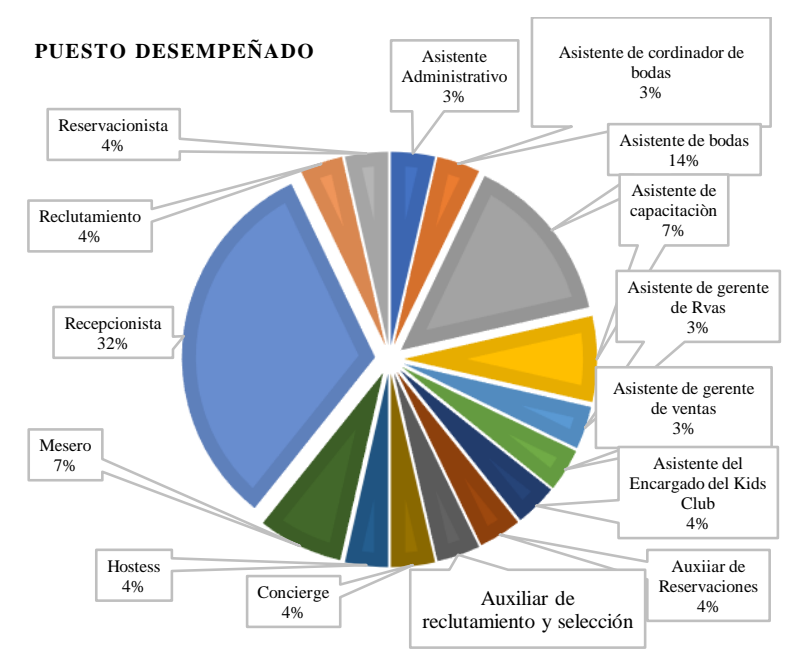

Gráfica 4 Puesto desempeñado

Fuente: Elaboración Propia

\section{- $\quad$ Factores nivel de inglés}

Dentro de este factor se analizaron dos enfoques, el primero relacionado a la percepción del alumno respecto al dominio del idioma inglés para posteriormente contrastar este dato con la certificación obtenida. Sobre la percepción que tiene el alumno del dominio de idioma inglés se observa que el $90 \%$ se ubica entre los niveles B1y B2 (ver gráfica 5).

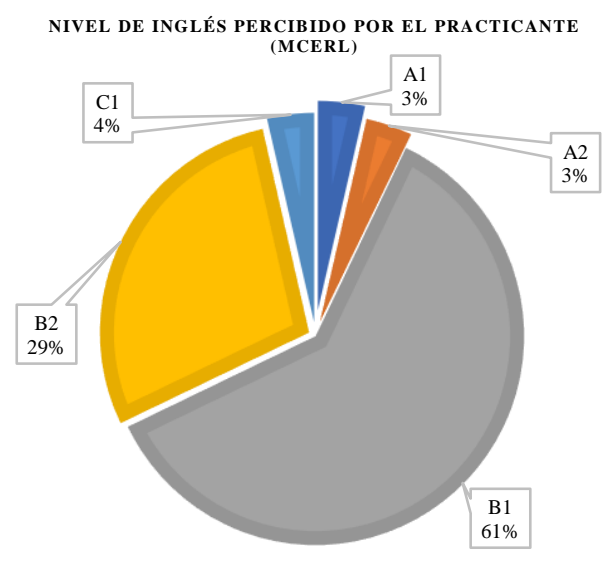

Gráfica 5 Nivel de Inglés percibido por el alumno Fuente: Elaboración Propia
Ahora bien, respecto al nivel de inglés certificado del alumno que se insertó a estadías se observa que el $75 \%$ se ubica entre los niveles B1 y B2, mientras que el $25 \%$ se encuentra entre los niveles A1 y A2 (ver gráfica 6).

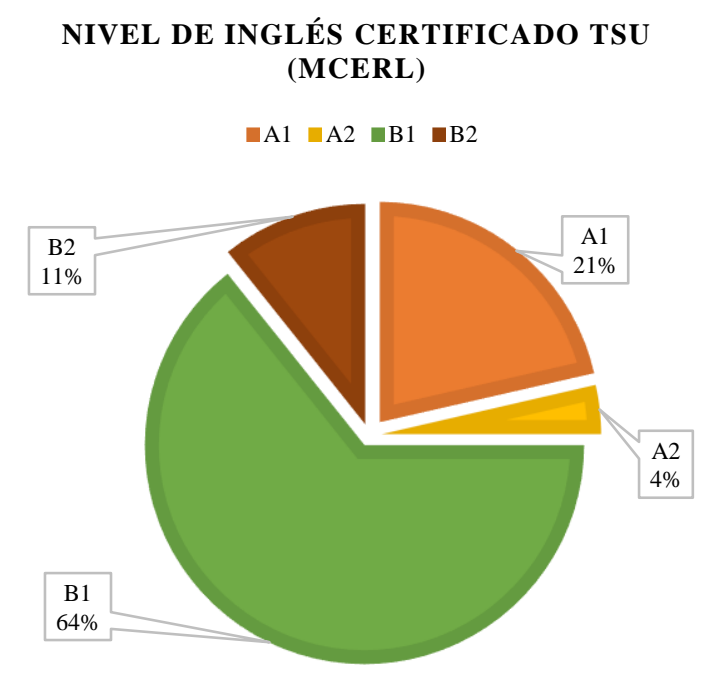

Gráfica 6 Nivel de Inglés certificado por el alumno al terminar TSU

Fuente: Elaboración Propia

\section{Comparativo de inserción a estadías entre modelo BIS y el tradicional}

Para poder hacer el comparativo entre el programa de competencias tradicional y el programa BIS se tomaron solo los factores sociográficos de género y los factores de inserción a los departamentos y puestos durante el proceso de estadía profesional.

\section{- $\quad$ Factor Demográfico}

Haciendo la comparación de género de la población objeto de estudio, se identifica que dentro del modelo tradicional predominaba el género masculino con un $59.40 \%$, mientras que en el modelo BIS, tanto hombres como mujeres tienen una representación del 50\% (ver gráfica $7)$.

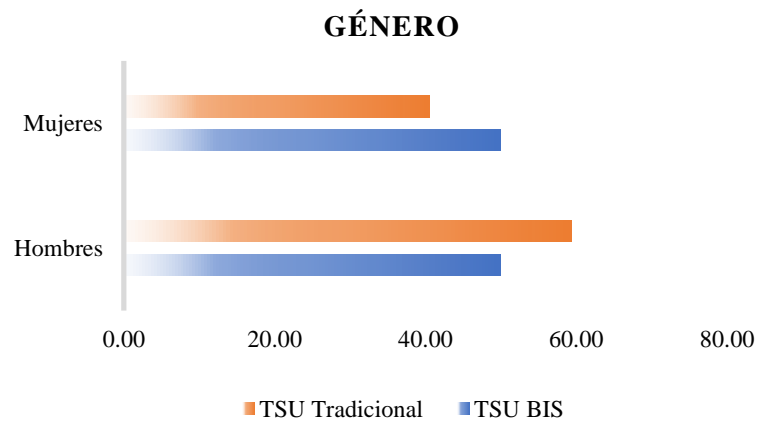

Gráfica 7 Comparativo de género entre modelo tradicional y BIS

Fuente: Elaboración propia

GÚADARRAMA-GÓMEZ, Irma, LARIOS-CALDERÓN, Aralí y BALDERAS-ELORZA, Carlos Raymundo. Comparativo de inserción a estadías del nivel TSU en Turismo de la UTRM entre el modelo BIS y tradicional. Revista de Ciencias de la Educación. 2019 
- Factor Departamento o puesto de trabajo

Para realizar el análisis de este factor, primero se clasificó la información de los departamentos según el área operativa (Front of the House) y administrativa (Back of the House), obtenida del estudio de Balderas, Guadarrama y Larios (2017). Cabe destacar que, para efecto de esta investigación se consideraron a los siguientes departamentos como parte del Front: recepción, reservaciones, concierge, servicio al cliente, actividades recreativas y relaciones públicas. Mientras que, para el Back se agruparon los siguientes: ama de llaves, recursos humanos, operaciones, marketing, ventas, capacitación, administrativa, calidad y desarrollo, grupos y convenciones, y alimentos y bebidas; en estas tres últimas desarrollando funciones administrativas.

Con los resultados del estudio del 2017 se identificó que el $62 \%$ de los alumnos realizaron sus prácticas profesionales en el Back. En contraparte, un 28.3\% las realizó en el Front. El $9.7 \%$ restante se insertó en otras áreas no especificadas.

Para la comparación de las áreas del Front y Back, se tomaron los resultados mencionados anteriormente y el factor de inserción al área departamental del Modelo BIS, en donde se observa que dentro del nuevo modelo hay una mayor inserción en el área de atención directa al cliente con un $69 \%$, en contraste con un $28.3 \%$, del modelo tradicional. Con respecto al Back, los datos se invierten, teniendo un $31 \%$ en contra de un $62 \%$ respectivamente. (ver tabla3)

\begin{tabular}{|l|r|r|r|}
\hline \multicolumn{1}{|c|}{ Modelo } & \multicolumn{1}{|c|}{ Front } & Back & Sin especificar \\
\hline Tradicional & $28.3 \%$ & $62 \%$ & $9.7 \%$ \\
\hline BIS & $69 \%$ & $31 \%$ & - \\
\hline
\end{tabular}

Tabla 3 Inserción en el Back y Front entre modelo BIS y Tradicional

Fuente: Elaboración Propia

Respecto a los departamentos y puestos en los que los alumnos se insertaron durante su periodo de prácticas, se identificó que en el modelo BIS el departamento con más practicantes adscritos a este fue recepción (29.63\%). Mientras que, en el modelo tradicional había más estudiantes realizando sus prácticas en el departamento de ama de llaves $(24.26 \%)$.
Cabe destacar que, de los alumnos del modelo BIS que forman parte de este estudio (primera generación), ninguno se insertó en este último departamento (ver gráfica 8).

\section{DEPARTAMENTOS DE INSERCIÓN}

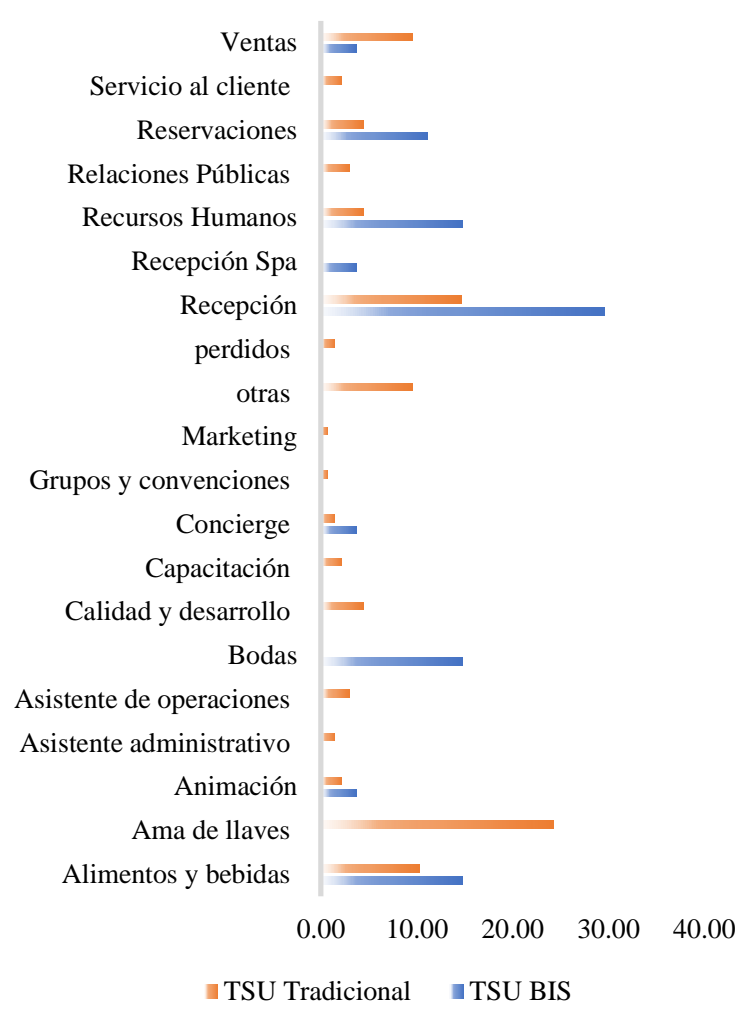

Gráfica 8 Comparativo de inserción departamental entre modelo tradicional y BIS

Fuente: Elaboración propia

\section{Conclusiones}

Las prácticas profesionales pueden ser vistas de diversas perspectivas, por un lado, son una herramienta que le permite al estudiante ganar experiencia dentro del campo laboral, por otra parte, a la Institución Educativa le permite identificar la efectividad de su programa educativo.

Considerando las áreas departamentales para realizar las prácticas profesionales, el comparativo permite identificar que los alumnos del modelo BIS han presentado un cambio considerable en la inserción de puestos, teniendo mayor incidencia aquellos que tiene trato directo con el cliente, a diferencia de los alumnos del programa tradicional que se insertaron más en áreas administrativas. Cabe destacar que, si bien el alumno puede mostrar interés en ocupar determinado puesto para realizar sus prácticas, es la empresa quien determina su ubicación con base en sus competencias. 
Según Balderas, Larios y Guadarrama (2017) respecto a la percepción que tenía el practicante sobre el nivel de dominio del idioma inglés en sus tres competencias (hablado, escrito y traducción); el alumno de TSU se aprecia con un dominio que no sobrepasaba el $50 \%$.

Ahora bien, con la inclusión de la UTRM al Modelo BIS, el nivel del idioma inglés se mide de acuerdo con el Marco Común Europeo de Referencia de Lenguas (MCERL) y el parámetro de medición es la certificación del idioma.

Tanto en el modelo tradicional como en él BIS se identifica que la percepción del conocimiento y dominio del idioma inglés es mayor al que se obtiene al momento de certificarse. Los alumnos al terminar el TSU deben tener nivel B1/B2 certificado. Sin embargo, el nivel general certificado ronda entre los niveles A2 y B1.

A los alumnos que no alcanzan la certificación se les permite continuar con los estudios, condicionados a obtener el nivel requerido en un tiempo determinado.

Los egresados de TSU modelo BIS están atendiendo la demanda de trabajadores calificados y cumplen más con los requerimientos del sector productivo. Sin embrago, no se puede afirmar que la migración al sistema bilingüe sea la causante principal de este cambio, se tendrá que hacer un estudio extensivo para asegurar la efectividad del programa y comprobar que existe una tendencia de inserción a los puestos del "Front".

\section{Referencias}

Álvarez-Gayou Jurgenson, J. L., Martín Camacho y López, S., Trejo García , C. Á., Olguín López, A., \& Pérez Jiménez, M. (2014). La investigación cualitativa. Xikua, Boletines Científico de la Escuela Superior de Tlahuelilpan, $\mathrm{s} / \mathrm{n}$.

Balderas Elorza, C. R., Larios Calderón , A., \& Guadarrama Gómez, I. (2017). Comparativo de inserción del proceso de estadías o prácticas profesionales de los niveles educativos de Técnico Superior Universitario en Turismo y Licenciatura en Gestión y Desarrollo Turístico. En H. P. Sergio Tobon Tobon (Autor), La gestión del Talento Humano en Latinoamérica: Análisis de Algunas Experiencias. ESTADOS UNIDOS DE AMÉRICA: Kresearch Corp.
CGUTyP. (2017). La experiencia del Modelo Bilingüe, Internacional y Sustentable de las Universidades Tecnológicas y Politécnicas . El Retoño: UT Retoño - Dirección Académica, México.

CGUTyP. (2018). Universidades Tecnológicas y Politécnicas Bilingües, Internacionales $y$ Sustentables. México DF.

Gómez Diaz de León , C., \& De León De la Garza , E. A. (2014). Método comparativo. Obtenido de Repositorio Institucional UANL. Universidad Autónoma de Nuevo León: http://eprints.uanl.mx/9802/1/Estudio\%20Comp arado.pdf

Hernández Sampieri, R., Fernández Collado , C., \& Baptista Lucio, P. (2014). Metodología de la Investigación . México : McGraw Hill Education.

Instituto Cervantes. (15 de 09 de 2017). Niveles cumunes de referencai: escala global. Obtenido de

https://cvc.cervantes.es/ensenanza/biblioteca_el e/marco/cap_03_01.htm

SEP. (17 de Diciembre de 2017). Universidades Tecnológicas y Politécnicas con Modelo BIS responden a demanda global de profesionistas de calidad. Obtenido de https://educacionsuperior.sep.gob.mx/comunica dos/2016/271216.html

Torres , F., \& Rojas , A. (jul de 2015). Revista SciELO. Obtenido de Problemas del Desarrollo : http://www.scielo.org.mx/scielo.php?script=sci _arttext\&pid=S0301-70362015000300041

Universidad Tecnológica de la Riviera Maya. (2019). Facebook UTRM. Obtenido de https://www.facebook.com/UTRivieraMaya.BI S/photos/a.10150101131055376/101568997575 70376/?type $=3 \&$ theater

wikipedia . (24 de 07 de 2019). Wikipedia. Obtenido de https://es.wikipedia.org/wiki/Pr\%C3\%A1ctica_ profesional 